\title{
The Management of Electric Power Harmonic
}

\author{
Tong $X \mathrm{u}^{1, \mathrm{a}}$ \\ ${ }^{1}$ north China Electric Power University, Beijing, 102206, China \\ ${ }^{a}$ xutong@ncepu.edu.cn
}

Keywords: Electric power, Harmonic, Compensation device, Filter

\begin{abstract}
The problem of electric power harmonic brought by nonlinear load in power grid has become more and more serious. The paper analyzes the main source and the causes of harmonics. The harm brought by electric power harmonic in electric equipment itself and power grid users are also be discussed. The paper demonstrates various measures to govern the harmonic in detail. The principle and structure of filter and harmonic compensation device are expounded in order to eliminate the damage in power gird caused by electric power harmonic.
\end{abstract}

\section{Introduction}

With the fast development of industry in our progressing society, diversification of electric machines and social modernization have been leveled up. Thus new-style electric and electronic devices are increasingly widespread using in industry, transportation and household appliances. Meanwhile, the increase of power grid loss caused by harmonic wave of nonlinearity load, occupies system capacity and reduces network efficiency, which results in voltage waveform distortion and loss of power quality. These abnormities may falsely activate or hold back relaying protection, then disturb regular operation or even extensive power failure. Harmonic wave can be enlarged dozens of times or more by system syntony,when it functions in power grid with capacitive components like reactive compensation capacitor, and lead to overvoltage damage to network devices.

Under ideal circumstance, supply voltage and current waveforms should both be sinusoidal wave. But it comes out that they are diverged in real power systems, by large amounts of power supply units and electric equipment possess a nonlinear impedance characteristic. They can be Fourier transformed into the sum of a DC value, a fundamental sinusoidal wave and a series of integer harmonics. The integer harmonic is actually disturbance variable, and is one of the main measuring indicators of power quality.

\section{Where Do Integer Harmonics Come From}

Integer harmonics no longer come from field current produced by transformers and iron core coils as they used to be. Nowadays, electric and electronic devices have become main sources of integer harmonics. These nonlinear loads are connected with power grid, and inject harmonic current into the network.

There are two basic sources of harmonic waves:

Nonlinear components contain electron tube or semiconductor, such as AC/DC converters, rectifiers, frequency converters on machines and electronic control units.

Nonlinear components contain ferromagnet and voltaic arc, such as: transformers, arc welding machines, fluorescent lamps and mutual inductors.

Why there is harmonic wave: 
Low quality power source: In a general way, power sources produce harmonic waves but usually not much.

Transmission and distribution system: In system, it is discharging arcs, transformers and core saturation that produce harmonic waves.

Electric equipment: the biggest harmonic wave source. $40 \%$ of the harmonic wave in PCT rectification of transformer substation is produced by rectification units. Frequency conversion devices not only produce integer harmonics, but also produce fraction-harmonics, due to phase control technology. Arc welding, gas discharge lamp, frequency conversion equipment all can bring harmonic wave to the power grid.

\section{Harm of Integer Harmonics}

Harm to the equipment. To the transformer: enhance core loss, copper loss and noise. To the motor: raise excess loss, lower the efficiency. To relay protection devices: cause malfunctions and rejections, inaccurate measure or system halted and messy code.

Harm to the User. With a large sum of frequency conversion equipment and electromagnetic devices connected in, power grids abound with integer harmonics. Among all kinds of harmonics, third harmonics take the largest amount. Harmonics whose frequency are multiples of three superposition at the central line. This enhance the central current, distort the voltage wave into peaked wave, and do harm to electric devices.

\section{The Management of Electric Power Harmonic}

With the growing seriousness of electric power harmonic pollution, international community formulates governance measures and related standards one after another. They set specific limitations to the electric power devices which cause pollution in order to limit overvoltage and the current distortion in power distribution system. The main measures to govern electric power harmonic are listed as follows.

Improve the Running State. Change certain operation connection schemes to reduce the electric power harmonic's production, superposition and amplification. In addition, reduce the possibility of producing harm and strengthen the monitor in running state. The running state of light load and high voltage can also be avoided.

Improve the Design of Electrical Equipment. In the design, manage harmonic to avoid producing resonance. In the design of reactive compensation, pay attention to avoid parallel resonant capacitor and system impedance. In addition, the main harmonics of 3, 5, 7odd harmonic should also be checked in case to the resonance in this class harmonic.

Install Harmonic Compensation Devices. Install the high-pressure dynamic reactive power compensation and filtering device on high voltage line.

Controller collects real-time voltage and current value of PT and CT. With the management of high precision meter chip to calculate the voltage, current, power factor, reactive power and other electric parameters of power grid. Besides, whether to switch capacitor is determined by single-chip microcomputer. Under a certain control measure, according to the threshold set in advance to judge whether the capacitor should be switched. If so, the controller will give instructions to vacuum contactor(QF) to accomplish an operation of capacitor.

As is shown in Fig.1, capacitor provides fixed capacitive reactive Qc. Compensation reactor determines the output perceptual reactive power QTCR of compensation electric reactor by current. The size of perceptual reactive power and capacitive reactive power phase offset. As long as the 
system reactive power can be done as follows: $Q n=Q v($ system required) $-Q C+Q T C R=$ constant (or zero). In this way, the power grid factor is a constant and voltage hardly fluctuates. The key point is to control the thyristor trigger angle to get the required electric current which flows through the compensation reactor. Thyristor converter device and control system can realize the function. Collect bus current reactive value and voltage value to compound reactive power value. Compared it with the value of constant reactive power to calculate the triggering angle size. Calculate required current flows through thyristor trigger device.

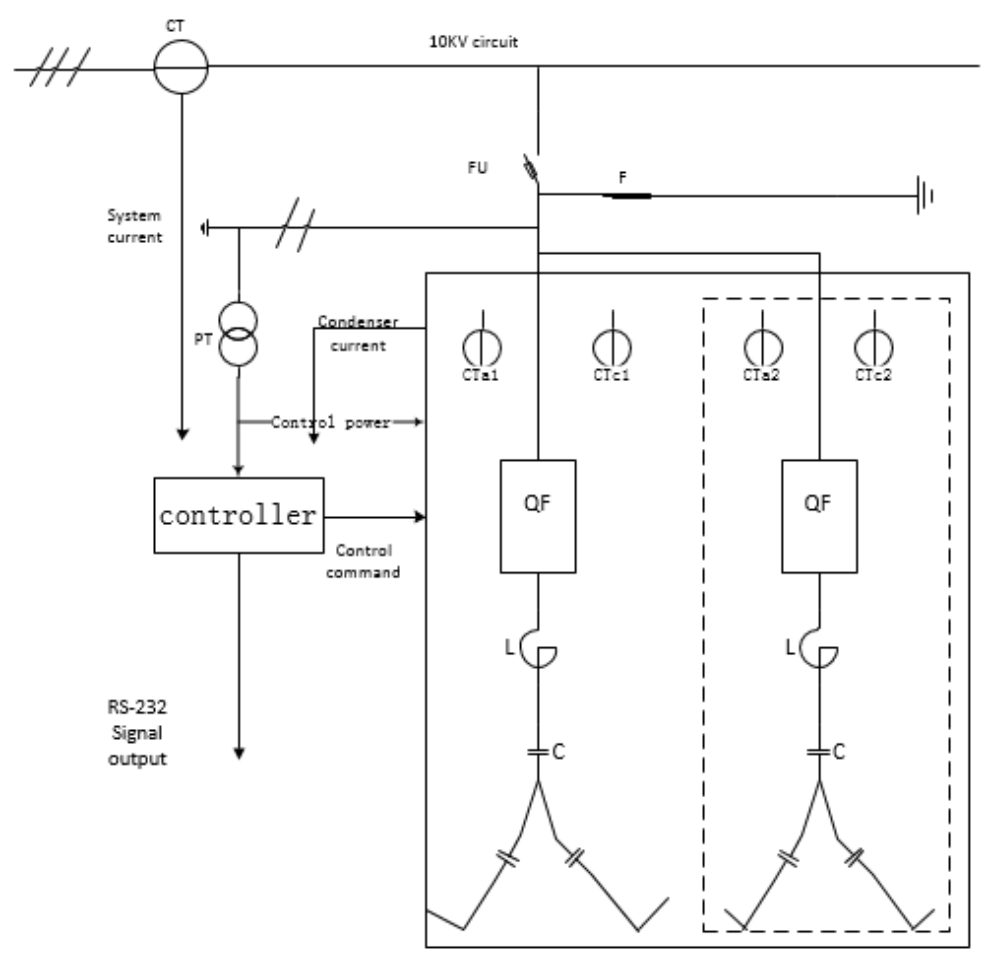

Fig.1 High-pressure dynamic reactive power compensation and filtering device installed

For asymmetric load, use split phase adjustment to eliminate negative sequence current and balance three-phase power gird. Cascade reactor in the capacitance compensation branch to suppress the amplification of harmonic current in power grid and increase the safety and reliability of power system. The device also has perfect protection functions. It has capacitor velocity fault protection, over-current protection, over-voltage protection, under-voltage protection, time-delay protection, phase break protection and protection functions such as limitation of action number per day.

Low voltage passive filter and dynamic reactive power compensation device.Low voltage passive filter and dynamic reactive power compensation device which is shown in Fig.2 are mainly used for $0.4 \mathrm{kV}, 0.66 \mathrm{kV}, 0.9 \mathrm{kV}$ voltage grade of power grid, such as 3, 5, 7, 11, 13 power and above of harmonic filter. According to the size of the load harmonic and reactive power, using thyristor switch to switch harmonic filter automatically to realize the nonlinear load to dynamically filter harmonic and compensate reactive power. It is especially suitable for the coexistence of large capacity and harmonic reactive power. Besides, the fixed harmonic represents a large proportion of loads. It has the features of rational design, good filtering effect, fast response and complete protection, stable and reliable work, etc. It can meet the rapid change of the load to the requirement of reactive power and harmonic. 


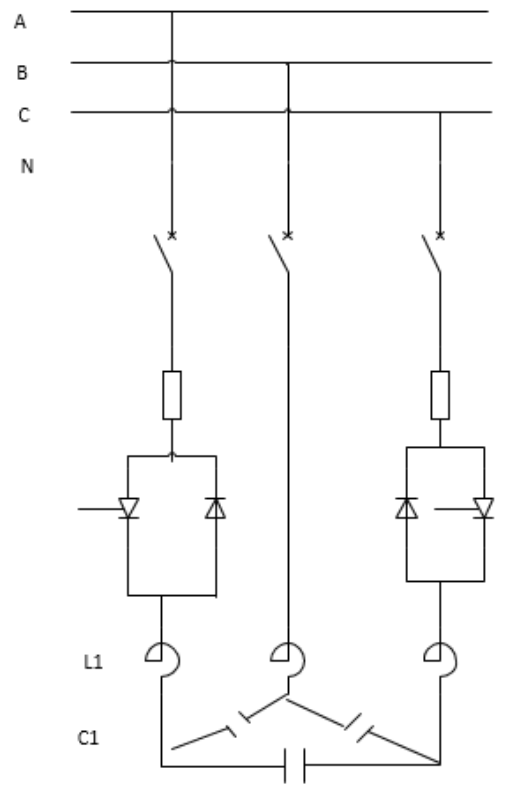

Fig.2Low voltage passive filter and dynamic reactive power compensation device.

As is shown in Fig.3, control system of equipment acquire circuit voltage, load current, output current, compensation system current and capacitor voltage signal by measuring circuit at first. Then using the measured signal to detect every harmonic component of load current and unbalanced weight. Considering the control must exist error, in order to improve the effect compensation, then check out every harmonic component of load current and unbalanced weight after compensation. Superimpose every harmonic component and unbalanced weight to conclude the output current reference signal of the device. In this way, the control system's digital signal processor can calculate inverter PWM control pulse according to the current reference signal. In addition, it drives inverter bridge device (IGBT) through the electronic switch to output corresponding every harmonic component and unbalanced component to realize compensation function.

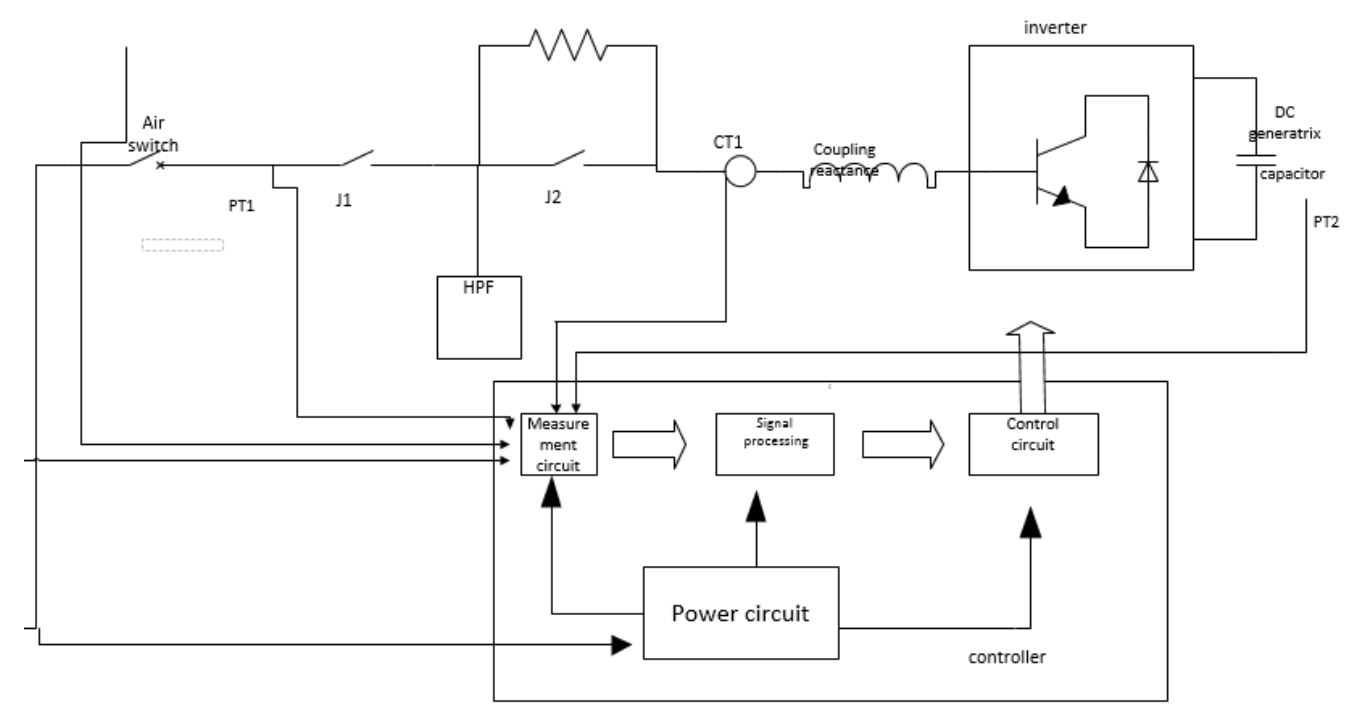

Fig.3 Low voltage active power filter device.

The device can filter every harmonic of 2 25 power in load current efficiently, so as to make the distribution network clean and efficient and meet the requirements of the harmonic in distribution network. The device can automatically identify the overall change of the load and the change of the 
load harmonic content to track and compensate quickly. The load change can be responded in 80us and full tracking compensation can be realized in $20 \mathrm{~ms}$. This device can eliminate the system imbalance caused by harmonic. If the capacity of equipment permits, the negative sequence and zero sequence components of compensation system can be set according to users. And reactive power can be compensated moderately. On the basis of ensuring to filter harmonic, the device improve the system imbalance effectively. Additionally, the device will not produce resonance with the grid and it can restrain the grid's own resonance within its capacity permission. This is what passive filter device can’t do.

\section{Conclusions}

As you can see, harmonics have already become a serious threat to the power grid, the equipment and the user. We can no longer ignore its harm but to deal with it immediately. A power filter can be used in harmonic current compensation, as well as in flicker suppression,system voltage stabilization and compensation of reaction power. We should devote to generalize power filter to improve the power grid function, and then the power grid will become more environment-friendly and sustainable

\section{References}

[1] Lascu C, Asiminoaei L, Boldea I, et al. High Performance Current Controller for Selective Harmonic Compensation in Active Power Filters[J]. IEEE Transactions on Power Electronics, 2007, 22(5):1826-1835.

[2] Luo A, Shuai Z, Zhu W, et al. Combined System for Harmonic Suppression and Reactive Power Compensation[J]. IEEE Transactions on Industrial Electronics, 2009, 56(2):418-428.

[3] Q. S. Wang, C. S. Kou, D. B. McDermott, et al. High-power harmonic gyro-TWT's. II. Nonlinear theory and design[J]. IEEE Transactions on Plasma Science, 1992, 20(3):163-169.

[4] Jou H L, Wu J C, Chang Y J, et al. A novel active power filter for harmonic suppression[J]. IEEE Transactions on Power Delivery, 2005, 20(2):1507-1513.

[5] Lascu C, Asiminoaei L, Boldea I, et al. High Performance Current Controller for Selective Harmonic Compensation in Active Power Filters[J]. IEEE Transactions on Power Electronics, 2007, 22(5):1826-1835. 